\title{
Priority Differentiation in Cognitive Radio Networks
}

\begin{abstract}
Cognitive radio networks rely on spectrum sensing performed in a collaborative manner by the cognitive nodes themselves. Priority differentiation in such a network can be accomplished through different scheduling policies, differentiated duration of mandatory spectrum sensing, or a combination of the two. This differentiation will affect not only packet delays, but also the accuracy of channel sensing and, by extension, the probability of collisions with primary user transmissions which will critically affect the operation of the network. In this paper we provide a probabilistic analysis of the interplay between priority differentiation and network performance, and investigate the resulting tradeoffs under different prioritization approaches.
\end{abstract}

\section{INTRODUCTION}

Priority differentiation in which different traffic classes experience different levels of service is often used to achieve the desired quality of service (QoS) in a wireless network [4]. Cognitive or opportunistic spectrum access networks pose unique challenges in the process of achieving the desired QoS [13]. In particular, the cognitive radio network must operate smoothly and with minimum interruptions caused by collisions with the transmissions of licensed primary users, which is accomplished through timely and accurate spectrum sensing which is often performed in collaboration of cognitive nodes with the network access point (coordinator) [19]. For reasons of cost and simplicity, cognitive nodes typically have a single radio which is used for both data communications and spectrum sensing. As the result, spectrum sensing interacts with data communications and, thus, affects the performance of the network. The problem is especially pronounced when the cognitive radio network employs traffic prioritization to achieve the desired QoS levels.

In this paper, we discuss the performance of data transmission in a cognitive radio network with collaborative spectrum sensing. The network accomodates multiple traffic classes with different priorities, which are differentiated through different service disciplines, the amount of spectrum sensing service, or a combination of the two.

In terms of service discipline, we consider 1-limited and exhaustive service - one for its simplicity, the other because it offers lower service delays at low to medium system load [9]. As data packets can't be transmitted during sensing or reception, the scheduling policies are actually gated with the moment of application for bandwidth.

In terms of spectrum sensing, we use a MAC protocol in which each packet transmission must be followed by mandatory spectrum sensing [12] and differentiate the traffic classes by the amount of sensing that the node of a specific traffic class has to provide.

Our analysis uses the tools of probabilistic analysis and renewal theory, and thus results in complete probability distribution for a number of important performance indicators such as packet waiting time and probability of collisions with primary user transmissions. As will be seen, the combination of differentiation mechanisms provide an effective way of controlling the performance of the network, but tradeoffs must be made between packet delays and collision probability. To the best of our knowledge, this is the first paper to investigate such a tradeoff in detail.

The paper is organized as follows: Section II presents an overview of related work. Section III gives more details about the operation of a frequency hopping CPAN piconet and describes the priority differentiation mechanism. Sections IV, $\mathrm{V}$, and VI provide the details of the analytical model of transmission and sensing, collisions, and packet waiting times. Section VII presents the performance data, while Section VIII concludes the paper and highlights some avenues for future research.

\section{RELATED WORK}

QoS provisioning and differentiation have been extensively studied in the context of different wireless paradigms, including cognitive networks.

A cross-layer approach that integrates spectrm sensing at the physical (PHY) layer with QoS-aware packet scheduling at the medium access control (MAC) layer has been proposed in [14]. Each node has two radios, one that enables it to listen to the dedicated control channel all the time, and another that is used in a cognitive manner to sense and dynamically use available channels. However, the cost of having two radios and the unavailability of a dedicated control channel renders this protocol infeasible in many applications.

The scheme proposed in [6] focuses on scheduling of uplink data transmissions with QoS provisioning, striving to provide an acceptable balance between system utilization, fairness, and delay constraints for individual secondary users, all the while respecting the requirement to minimize the interference to primary users.

The analysis in [8] explores the ability of cognitive radio to provide virtual networks that use residual spectrum that is not utilized by wireless network operators. SUch networks are shown to offer sufficient capacity to support additional QoS traffic with acceptable performance.

The game-theoretic approach described in [2] focuses on a cognitive network that uses negotiated secondary access in which the interactions between the primary and the secondary systems take place on a dedicated signaling channel; a centralized resource allocation scheme is then used to meet the QoS requirements for both primary and secondary users.

A queueing network model based on $\mathrm{M} / \mathrm{G} / 1$ queues with preemptive resume priority that analyzes QoS performance of various spectrum management techniques has been described 


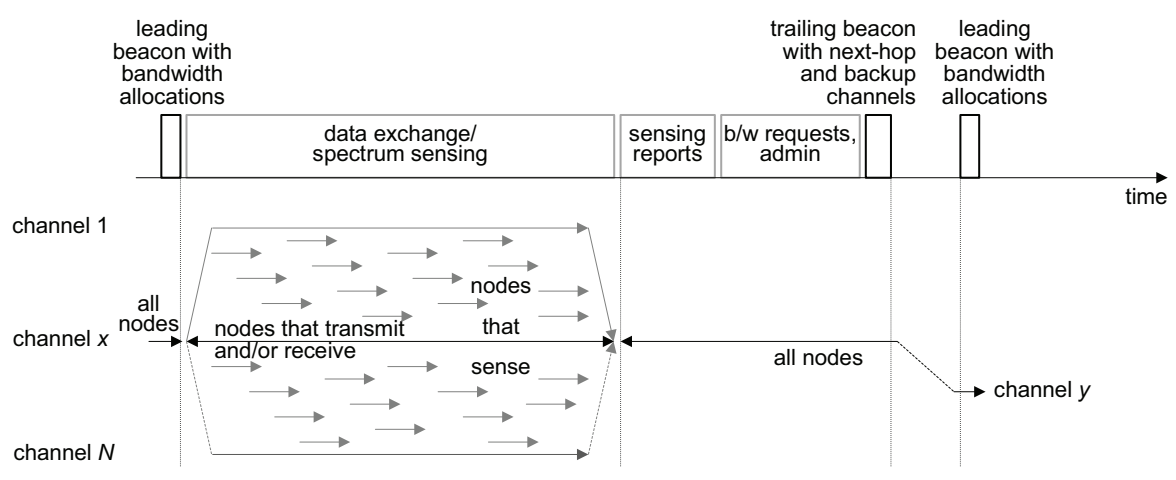

Fig. 1. The format of a superframe and operation of different nodes during the superframe.

in [18]. However, the analysis focuses on the difference between reactive and proactive spectrum decision model and their impact on QoS, similar to the analysis in [17], rather than on the manner in which the desired QoS can be achieved.

The work presented in [5] describes a MAC protocol that integrates spectrum sensing and QoS provisioning. In this scheme, the cognitive (secondary) nodes are not required to have two independent radios; however, it is assumed that only a small subset of working channels is sensed in a given transmission slot, and nodes are free to choose the best channel in each slot. Furthermore, all communications involve the base station through separate downlink and uplink packet transmissions.

Differentiation between real- and non-real-time users was considered in [16], where modifications were made to the CSMA/CA mechanism, including a combination of contention-based and contention-free access, in order to cater to the requirements of the cognitive network operating in the same frequency band as the number of licensed (primary) transmitters. A similar approach in [1] gives priority to real time users through allocating a certain number of channels for their exclusive use, while the remaining channels are allocated to non-real-time users as (and if) available.

The distributed MAC protocol described in [3] differentiates between several classes of multimedia traffic through different sensing periods. Namely, higher priority traffic is assigned shorter intervals between channel sensing events which results in different probability that an idle channel will be identified and immediately used. However, the differentiation thus obtained may be difficult to control due to randomness of primary user activity patterns.

As can be seen, while the issue of QoS provisioning has received a lot of attention, the interplay between priority differentiation and cognitive network performance, in particular the probability of collisions with primary user transmissions, is still largely unexplored, which was the motivation for our work reported in this paper.

\section{NETWORK OPERATION AND SERVICE DIFFERENTIATION POLICIES}

We consider the problem of priority differentiation in the context of a cognitive radio network which uses a MAC protocol with transmission tax [12].

A cognitive radio network, hereafter referred to as piconet, consists of a number of ordinary nodes and a dedicated coordinator node. To facilitate communications, the coordinator emits beacon and trailer frames that delineate the superframe, as shown in Fig. 1. Each superframe takes place on a single channel from the working channel set, with successive superframes taking place on channels chosen dynamically so as to avoid collisions with primary (licensed) user activity.

Different portions (sub-frames) of the superframe are dedicated to different data transmission and administrative activities. Transmission slots are requested during the reservation sub-frame, and subsequent allocations are announced in the leading beacon at the beginning of next superframe.

We assume that there are $m$ traffic classes in the network, where the class index corresponds to class priority in descending order (i.e., class 1 has the highest, class $m$ the lowest priority). Each traffic class $i=1 \ldots m$ has $M_{i}$ nodes, for a total of $M=\sum_{i=1}^{m} M_{i}$ nodes in the piconet.

Priority is used to determine the order and duration of medium access as well as the duration of spectrum sensing. Higher priority traffic will get allocated before lower priority later. Furthermore, higher priority traffic is scheduled using exhaustive service, in which a node that requests transmission with $n$ packets in the buffer will be granted time slots to transmit all $n$ packets, while lower priority traffic is scheduled using 1-limited policy, in which a node will be granted single packet transmission.

Transmissions from nodes of a single priority class are scheduled in a round robin fashion. Namely, the coordinator keeps track of the last node serviced in the previous superframe, and assigns transmission slots beginning from the first higher address. Allocation includes as many requests as can fit into one superframe; requests that can't be granted in the current superframe are deferred to the next one. Once all requests from a given priority class have been serviced, coordinator schedules the next lower priority class and so on.

Upon transmission, the node will be instructed by the coordinator to perform sensing for $k_{p}^{(i)} n$ subsequent superframes, where $k_{p}^{(i)}$ is the penalty coefficient for traffic class $i$ and $n$ is the number of transmitted packets $(n=1$ for 1-limited 


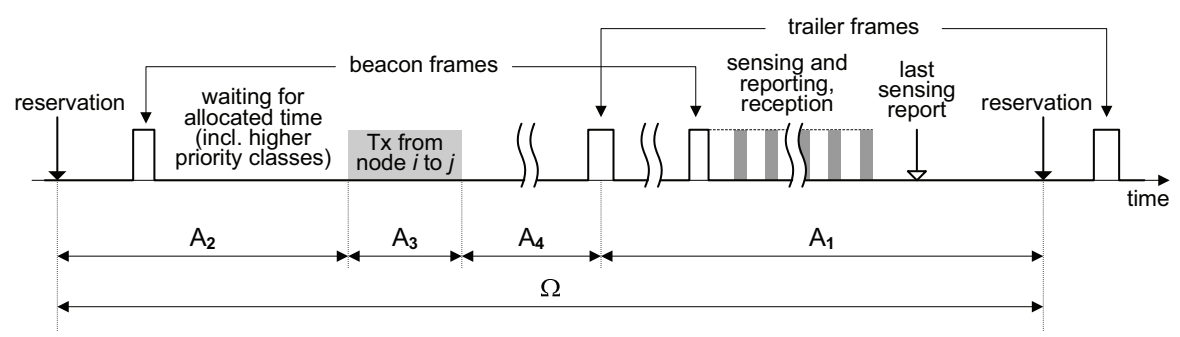

Fig. 2. Distribution of arrivals during service cycle.

policy). The choice of penalty coefficient can be used to further differentiate the traffic classes by allowing higher priority classes to spend less time in sensing.

Sensing reports are sent to the coordinator during the reporting sub-frame; if the sensing duty spans more than one superframe, reports are to be sent in each superframe, which ensures collision-free operation of the piconet. In practice, coordinator should instruct the node to start sensing when $k_{p}^{(i)} n$ reaches an integer value.

Sensing reports are used by the coordinator to determine the next-hop and backup channels [12]; these are announced in the trailer frame at the end of the superframe. All nodes must listen to both beacon and trailer frames so as to receive the administrative information contained therein, but also for synchronization purposes.

Packets that arrive during sensing are stored but not transmitted - the node can request new bandwidth allocation only upon finishing its current round of sensing duty. (In other words, both scheduling policies correspond to gated policies with vacations.) If the node has no packets to send upon finishing its sensing duty, it immediately launches another sensing cycle which ensures continued operation of the sensing mechanism during periods with little traffic. Sensing can be temporarily suspended in order to receive a packet from another node, and resumed in the following superframe.

\section{Modeling The MAC Algorithm}

The timing diagram showing the operation of a node is shown in Fig. 2. As can be seen, packet transmission cycle includes waiting for the reservation sub-frame, waiting for the transmission slot, actual transmission, sensing and synchronization with the beacon. Node transmission time is randomly positioned with respect to the beacon and the control and reservation sub-frames that immediately precede the beacon and, typically, have fixed duration. The distance between the end of a transmission and next beacon (which includes control and reservation sub-frames) is referred to as the beacon synchronization time.

For simplicity, all times are expressed as multiples of the basic sensing slot, so that the superframe length is $s_{f}$ slots and the packet size (assumed constant) is $k_{d}$ slots, while the acknowledgment lasts for one slot. Let the probability generating function (PGF) for the packet size, including acknowledgment, be $b(z)=z^{k_{d}+1}$ with a mean value of $\bar{b}=k_{d}+1$. Since the probability distribution of packet time with acknowledgment
TABLE I

PGFS FOR ARRIVAL PROCESSES

\begin{tabular}{lcc}
\hline period & PGF & mass probabilities \\
\hline waiting for allocated time (includes & $A_{2}^{(i)}(z)$ & $a_{2, k}^{(i)}$ \\
busy periods of higher priority classes) & & $A_{3}^{(i)}(z)$ \\
data transfer for target node & $A_{1}^{(i)}(z)$ & $a_{3, k}^{(i)}$ \\
sensing period with penalty & $A_{0}^{(i)}(z)$ & $a_{1, k}^{(i)}$ \\
sensing for a single superframe & $A_{4}^{(i)}(z)$ & $a_{4, k}^{(i)}$ \\
synchronization with the beacon & $A_{s}^{(i)}(z)$ & $a_{s, k}^{(i)}$ \\
single packet transmission & & \\
\hline
\end{tabular}

is discrete, its Laplace-Stieltjes transform (LST) is obtained by replacing variable $z$ with $e^{-s}$, i.e., $b^{*}(s)=e^{-s\left(k_{d}+1\right)}$. Packets arrive at the class $i$ node according to a Poisson process with arrival rate $\lambda_{i}$. Offered load at the class $i$ node is $\rho_{i}=\lambda_{i} \bar{b}$. We assume that all nodes have buffers of infinite capacity.

Let us now find the probability distribution of the number of packets in node buffer upon return from sensing. Let mass probabilities for $k$ packets in the traffic class $i$ node be $q_{j}^{(i)}$, and let the corresponding PGF for the number of packets queued be denoted with $Q^{(i)}(z)$.

To arrive at this PGF for exhaustive and 1-limited service classes, we need a precise characterization of periods defined in the MAC protocol. The PGFs for the arrival processes for those periods are summarized in Table I. In more compact notation, PGF for the arrival process between two successive applications for transmissions with non-empty buffer is $A^{(i)}(z)=A_{1}^{(i)}(z) A_{2}^{(i)}(z) A_{3}^{(i)}(z) A_{4}^{(i)}(z)$, and its mass probabilities are $a_{k}^{(i)}$. (If the node returns from sensing with an empty buffer, it undertakes additional sensing duty.) Balance equations for the exhaustive (first) and 1-limited (second) service policy, respectively, are

$$
\begin{aligned}
& q_{k}^{(i)}=q_{0}^{(i)} a_{1, k}^{(i)}+\sum_{j=1}^{\infty} q_{j}^{(i)} a_{k}^{(i)} \\
& q_{k}^{(i)}=q_{0}^{(i)} a_{0, k}^{(i)}+\sum_{j=1}^{k+1} q_{j}^{(i)} a_{k-j+1}^{(i)}
\end{aligned}
$$

By multiplying left- and right-hand sides of (1) and (2) and 
summing them from 0 to $\infty$, we obtain respective PGFs as

$$
\begin{aligned}
& Q^{(i)}(z)=q_{0}^{(i)} A_{1}^{(i)}(z)+\left(1-q_{0}^{(i)}\right) A^{(i)}(z) \\
& Q^{(i)}(z)=\frac{q_{0}^{(i)}\left(z A_{1}^{(i)}(z)-A^{(i)}(z)\right.}{z-A^{(i)}(z)}
\end{aligned}
$$

Probability $q_{0}^{(i)}$ can be found from the fact that $q_{0}^{(i)}=$ $Q^{(i)}(0)$, for the first case , and from normalization condition, for the second case:

$$
\begin{aligned}
& q_{0}^{(i)}=\frac{A^{(i)}(0)}{1-A_{1}^{(i)}(0)+A^{(i)}(0)} \\
& q_{0}^{(i)}=\frac{1-A^{\prime(i)}(1)}{1+A_{1}^{\prime(i)}(0)-A^{\prime(i)}(1)}
\end{aligned}
$$

where $A^{\prime}(i)(1)=\overline{A^{(i)}}$.

In both cases, the service period begins when there is at least one packet in the buffer. PGF for the number of packets when a request for bandwidth is made, is

$$
\Omega^{(i)}(z)=\sum_{k=1}^{\infty} q_{k}^{(i)} z^{k}=\frac{Q^{(i)}(z)-q_{0}^{(i)}}{1-q_{0}^{(i)}}
$$

where, of course each traffic class will have a distinct probability distribution expressed through its PGF $Q^{(i)}(z)$.

\section{A. Duration of the transmission period}

For classes with exhaustive scheduling duration of transmission of node from class $i$ has the PGF $S^{(i)}(z)=\sum_{k=1}^{\infty} s_{k}^{(i)} z^{k}$. Since we know the number of packets in the queue at the moment for bandwidth application, the transmission period can be expressed as

$$
\begin{aligned}
& S^{(i)}(z)=\Omega^{(i)}(b(z)) \\
& S^{(i)}(z)=b(z)
\end{aligned}
$$

with the mean value of $\bar{S}=\bar{\Omega} \bar{b}$ and $\bar{S}=\bar{b}$ for the exhaustive and 1-limited service, respectively.

After packet transmission, the node needs to wait for the beacon transmitted by the coordinator in order to learn about the next and backup channels, as shown in Fig. 1. This waiting time is residual superframe time with respect to a random point in the superframe, and its LST is $R^{*}(s)=\left(1-e^{-s \cdot s_{f}}\right) /(s$. $\left.s_{f}\right)$, while the number of packet arrivals to the node buffer during that time has the PGF of

$$
A_{4}^{(i)}(z)=R_{-}^{*}\left(\lambda_{i}-\lambda_{i} z\right)
$$

\section{B. Sensing periods}

Nodes from class $i$ perform sensing for the period proportional to the class penalty coefficient and number of packets transmitted during the busy period. The PGF for the time node spent in sensing is

$$
\begin{aligned}
& V^{(i)}(z)=\sum_{k=1}^{\infty} q_{k}^{(i)} z^{k k_{p}^{(i)} s_{f}}=\Omega^{(i)}\left(z^{k_{p}^{(i)} s_{f}}\right) \\
& V^{(i)}(z)=z^{k_{p}^{(i)} s_{f}}
\end{aligned}
$$

with mean values of $\overline{V^{(i)}}=k_{p}^{(i)} s_{f} \overline{\Omega^{(i)}}$ and $\overline{V^{(i)}}=k_{p}^{(i)} s_{f}$ for exhaustive and 1-limited service, respectively.

The number of packet arrivals to the node during a single vacation period has the PGF of

$$
A_{1}^{(i)}(z)=V^{(i) *}\left(\lambda_{i}-\lambda_{i} z\right)=\sum_{k=0}^{\infty} a_{1, k}^{(i)} z^{k}
$$

which is independent of the service policy [15], and the probability of zero packet arrivals is $a_{1,0}^{(i)}=V^{(i) *}\left(\lambda_{i}\right)$.

Probability distribution of the total duration of sensing periods is given by

$$
I^{(i)}(z)=\frac{V^{(i)}(z)\left(1-q_{0}^{(i)}\right)}{1-V^{(i)}(z) q_{0}^{(i)}}
$$

\section{Waiting for service}

A class $i$ node has to wait until all nodes from higher priority classes, as well as nodes from the same priority but with IDs lower than its own that have had packets, have been serviced. Let the time between two successive transmission opportunities for a node be denoted as the class $i$ cycle time; its LST is

$$
C^{(i) *}(s)=\prod_{j=1}^{i-1} C^{(j) *}(s)\left(\left(1-P_{r}^{(i)}\right)+P_{r}^{(i)} S^{(i)} *(s)\right)^{M_{i}}
$$

where $S^{(i)} *(s)$ was defined in (8), while $P_{r}^{(i)}$ denotes the probability that the node is involved in the current cycle. Mean duration of the class $i$ cycle is $\overline{C^{(i)}}=-C^{*^{\prime}}(0)=$ $-\left.\frac{d C^{(i)} *(s)}{d s}\right|_{s=0}=\rho_{i} M_{i} \overline{S^{(i)}}$

After applying for bandwidth, node from class $i$ has to wait for full cycle of higher priority traffic as well as for all nodes from the same class that have smaller IDs. Under Poisson packet arrivals, a random number of class $i$ nodes participate in each cycle. As the node ID is randomly positioned within the set of IDs of nodes serviced in the current cycle, the latter period is, in fact, the elapsed cycle time [7] which can be described with

$$
C_{-}^{(i) *}(s)=\frac{1-C^{(i) *}(s)}{s \overline{C^{(i)}}}
$$

Its mean value is $\overline{C_{-}^{(i)}}=\frac{C^{(i)(2)}}{2 \bar{C}}$, where $C^{(2)}=C^{*^{\prime \prime}}(0)=$ $\left.\frac{d^{2} C^{(i) *}(s)}{d s^{2}}\right|_{s=0}$ denotes the second moment of the cycle time.

The number of packet arrivals during the time spent waiting for the round-robin service has the PGF of

$$
A_{2}^{(i)}(z)=\prod_{j=1}^{i-1} C^{(j) *}\left(\lambda_{i}-\lambda_{i} z\right) C_{-}^{(i) *}\left(\lambda_{i}-\lambda_{i} z\right) .
$$

The number of packets that arrive to the node during transmission time has the PGF of

$$
A_{3}^{(i)}(z)=S^{(i) *}\left(\lambda_{i}-\lambda_{i} z\right) .
$$




\section{Probability that a node is involved in the current cycle}

A node is involved in the current cycle when it is either transmitting or waiting for its turn to transmit, which occurs with the probability

$$
P_{r}^{(i)}=\frac{\overline{S^{(i)}}+\sum_{j=0}^{i-1} \overline{C^{(j)}}+\overline{C_{-}^{(i)}}}{\overline{S^{(i)}}+\sum_{j=0}^{i-1} \overline{C^{(j)}}+\overline{R_{-}^{(i)}}+\overline{C_{-}^{(i)}}+\overline{I^{(i)}}}
$$

By combining (5) to (19) we obtain the probability distribution of the number of class $i$ packets in the buffer upon return from sensing. Using those distributions for all traffic classes, all other performance parameters can be derived.

\section{COLLISIONS WITH PRIMARY SOURCE TRANSMISSION}

Since higher priority traffic classes transmit earlier in the superframe, they may be expected to experience fewer collisions with primary source transmissions. Collision probability has two components: one is caused by errors in the channel table maintained by the coordinator, which occur when the primary source changes the state between two sensing events; this probability is significant when the number of sensing nodes is small compared to the number of channels. The other component is caused by the onset of primary source activity during an ongoing superframe.

In order to evaluate this effect we assume that the piconet uses $N$ frequency channels, each of which is intermittently occupied by an independent primary source whose onset and OFF periods can be described with probability distribution functions (pdf-s) $t_{o n}(x)$ and $t_{o f f}$ respectively. Their mean values are $\overline{T_{o n}}$ and $\overline{T_{o f f}}$, respectively; the total cycle time of primary source has mean value $\overline{T_{c y c}}=\overline{T_{o n}}+\overline{T_{o f f}}$; and the activity factor of primary source is $p_{o n}=\frac{\overline{T_{o n}}}{T_{c y c}}$.

The time from the moment of arrival of the piconetq to the channel, which is a random point in the idle channel period, until the onset of channel activity is residual (channel) idle time. The pdf of this time is proportional to the probability that idle time is larger than some value $y$ scaled to the mean idle time, i.e., $d(y)=\frac{\int_{z=y}^{\infty} t_{o f f}(z) d z}{\overline{T_{o f f}}}$. PDF for residual idle time on the channel is, then, $D(x)=\int_{0}^{x} d(y) d y$.

\section{A. Probability that the coordinator has obsolete information}

Probability that a node from traffic class $i$ will be active in spectrum sensing can be calculated as

$$
P_{s}^{(i)}=\frac{\overline{I^{(i)}}}{\overline{S^{(i)}}+\sum_{j=0}^{i-1} \overline{C^{(j)}}+\overline{R_{-}^{(i)}}+\overline{C_{-}^{(i)}}+\overline{I^{(i)}}}
$$

The, the probability distribution for the number of nodes simultaneously involved in sensing can be described with a PDF of

$$
\Xi(z)=\prod_{i=1}^{m} \sum_{j=0}^{M_{i}}\left(\begin{array}{c}
M_{i} \\
j
\end{array}\right) P_{s}^{(i), j}\left(1-P_{s}^{(i)}\right)^{M_{i}-j} z^{j}=\sum_{n=0}^{M} \xi_{n} z^{n}
$$

where $\xi_{n}$ denotes mass probability that $n$ nodes from all classes are involved in spectrum sensing. (The coordinator can take part in spectrum sensing during the data exchange/sensing sub-frame.)

We assume that the sensing node randomly choose channels to sense among the $N-1$ channels with equal probability $P_{n}=1 /(N-1)$ (the channel used by the current superframe does not need to be sensed, of course). Let the period between two consecutive sensing events be denoted with $d$. Then, considering (21), we can derive the PGF for the time period between two consecutive sensing events on the same channel as

$$
\begin{aligned}
H(z)= & \xi_{0} \sum_{k=1}^{\infty} P_{n}\left(1-P_{n}\right)^{k-1} z^{k} d+\sum_{\min (M, N-2)+1}^{M} z^{d} \\
& +\sum_{l=1}^{\min (M, N-2)} \xi_{l} \sum_{k=1}^{\infty} l P_{n}\left(1-l P_{n}\right)^{k-1} z^{k} d
\end{aligned}
$$

This result enables us to find the duration of inaccurate channel state and calculate the probability $p_{s}$ of having inaccurate channel state in the channel table, esp. the case when a busy channel is recorded as idle. The derivation is omitted for space limitations; it can be found following the steps outlined in [11]. Note that $p_{s}$ is common to all traffic classes, even though their contribution to the sensing process may differ.

\section{B. Probability of collision for traffic class $i$}

Second component is the probability that the channel will become active during a node of traffic class $i$ transmits. Higher traffic classes will experience fewer collisions than the lower priority ones, since the former transmit sooner (i.e., closer to the beacon frame). Since the piconet arrival to an idle channel is random point in idle channel time, collision probability for traffic class $i$ can be calculated as the probability that residual idle channel time is shorter than the superframe duration:

$$
P_{c}^{(i)}=\int_{x=0}^{\infty}\left(D\left(x+\sum_{j=1}^{i} \overline{C^{(j)}}+\overline{C_{-}^{(i)}}+\overline{S^{(i)}}\right)-D(x)\right) d(x) d x
$$

The total collision probability is, then,

$$
P_{C o l}^{(i)}=P_{c}^{(i)}+p_{s}
$$

\section{PACKET ACCESS DELAY}

Under 1-limited service, the transmission from a class $i$ node will involve only the packet that was found at the head of the node buffer at the time of the bandwidth request; all other packets in the buffer, as well as those that have arrived since that request, will be serviced in future transmission cycles. Then, the PGF for the number of packets left after departing packet is

$$
L^{(i)}(z)=\frac{\Omega^{(i)}(z) A_{2}^{(i)}(z) A_{3}^{(i)}(z)}{z}
$$

On the other hand, under exhaustive service, all $L_{x}^{(i)}$ packets that were in the buffer at the time of the bandwidth request 
will be serviced; the $A_{s, j}$ that arrive during the service of the $j$-th packet will be serviced in future service cycles. Given the MAC priority structure and round robin scheduling within a given priority class, the number of packets to be served in following busy period is

$$
L_{n}^{(i)}=L_{x}^{(i)}+A_{2}^{(i)}+A_{s, 1}^{(i)}+A_{s, 2}^{(i)}+\ldots+A_{s, n}^{(i)}-n
$$

Let $Q^{(i)}(z)=\sum_{k=1}^{\infty} q_{k}^{(i)} z^{k}$ denote the PGF for the number of packet transmission slots requested from the coordinator. After multiplying the balance equations (26) by $z^{k} \mathrm{~m}$ and summing them for $k=1 \ldots \infty$, and upon additional scaling, we obtain the PGF for the number of packets left in the class $i$ buffer after $n$-th departing packet as

$$
L_{n}^{(i)}(z)=\frac{A_{2}^{(i)}(z) A_{s}^{(i)}(z)^{n} \sum_{k=n}^{\infty} q_{k}^{(i)} z^{k}}{z^{n} \sum_{k=n}^{\infty} q_{k}^{(i)}}
$$

Note that $L_{n}^{(i)}(1)=1$ which indicates that $L_{n}^{(i)}(z)$ is indeed a probability distribution.

We can now obtain the PGF for the number of packets left after any departing packet as

$$
\begin{aligned}
L^{(i)}(z) & =\sum_{n=1}^{\infty} \frac{\sum_{k=n}^{\infty} q_{k}^{(i)}}{\overline{Q^{(i)}}} L_{n}^{(i)}(z) \\
& =\frac{A_{2}^{(i)}(z)}{\overline{q^{(i)}}} \sum_{n=1}^{\infty} \sum_{k=n}^{\infty} q_{k}^{(i)} z^{k}\left(\frac{A_{s}^{(i)}(z)}{z}\right)^{n} \\
& =\frac{A_{2}^{(i)}(z)}{\overline{Q^{(i)}}} \sum_{k=1}^{\infty} q_{k}^{(i)} z^{k} \sum_{n=1}^{k}\left(\frac{A_{s}^{(i)}(z)}{z}\right)^{n} \\
& =\frac{A_{2}^{(i)}(z)}{\overline{Q^{(i)}}} \sum_{k=1}^{\infty} q_{k}^{(i)} z^{k} \frac{\left(\frac{A_{s}^{(i)}(z)}{z}\right)^{k+1}-\frac{A_{s}^{(i)}(z)}{z}}{\frac{A_{s}^{(i)}(z)}{z}-1} \\
& =\frac{A_{s}^{(i)}(z) A_{2}^{(i)}(z)}{\overline{Q^{(i)}}} \frac{Q^{(i)}(A(z))-Q^{(i)}(z)}{A_{s}^{(i)}(z)-z}
\end{aligned}
$$

Since $A_{s}^{(i)}(z)=b^{*}\left(\lambda_{i}-\lambda_{i} z\right)$, (28) can be written as

$L^{(i)}(z)=A_{2}^{(i)}(z) \frac{\left(Q^{(i)}\left[b^{*}\left(\lambda_{i}-z \lambda_{i}\right)\right]-Q^{(i)}(z)\right) b^{*}\left(\lambda_{i}-z \lambda_{i}\right)}{\overline{Q^{(i)}}\left[b^{*}\left(\lambda_{i}-z \lambda_{i}\right)-z\right]}$

with mean value of $\overline{L^{(i)}}=\overline{A_{2}^{(i)}}+\rho_{i}+\frac{\left(1+\rho_{i}\right) Q^{(i)(2)}(1)}{2 \overline{Q^{(i)}}}$, where $Q^{(i)(2)}(1)=\left.\frac{d^{2} Q^{(i)}(z)}{d z^{2}}\right|_{z=1}+\overline{Q^{(i)}} Q^{(i)(2)}(1)$ denotes the second moment for traffic class $i$.

Since $\rho_{i}$ refers to the packet currently in service by using Little's theorem, mean waiting time of class $i$ packet is $\overline{W_{i}}=$ $\overline{L^{i}} / \lambda_{i}$.

Probability distribution of waiting time in the node buffer can be found by observing that the number of packets left after the departing packet is equal to the number of packets which have arrived to the queue while target packet was in the system. Therefore,

$$
L^{(i)}(z)=W^{(i) *}\left(\lambda_{i}-\lambda_{i} z\right) b^{*}\left(\lambda_{i}-\lambda_{i} z\right)
$$

By using the substitution $s=\lambda_{i}-\lambda_{i} z$, we obtain LST of the packet waiting time for 1-limited and exhaustive policies, respectively, as

$W^{(i) *}(s)=A_{2}^{(i)}\left(1-\frac{s}{\lambda_{i}}\right) \frac{\lambda_{i}\left(Q^{(i)}\left[b^{*}(s)\right]-Q^{(i)}\left(1-\frac{s}{\lambda_{i}}\right)\right)}{\overline{Q^{(i)}}\left[\lambda_{i} b^{*}(s)-\lambda_{i}+s\right]}$

$W^{(i) *}(s)=\frac{\lambda_{i} A_{2}^{(i)}\left(1-\frac{s}{\lambda_{i}}\right) \Omega_{2}^{(i)}\left(1-\frac{s}{\lambda_{i}}\right)}{\lambda_{i}-s}$

Mean value of waiting time is, then, $\overline{W^{(i)}}=\left.\frac{d W^{(i) *}}{d s}\right|_{s=0}=$ $\frac{\left(1+\rho_{i}\right) Q^{(i)(2)}(1)}{2 \lambda_{i} \overline{Q^{(i)}}}$

\section{ViI. Performance eVAluation}

To obtain performance parameters we have solved the system of equations (8) to (10) using Maple 16 by Maplesoft, Inc. [10].

We have considered a piconet with the coordinator and two traffic classes with $M_{1}=M_{2}=9$ nodes each. Packet arrival rate was varied simultaneously for both traffic classes between $\lambda_{1}=\lambda_{2}=0.001$ and 0.005 packets per node per slot, for 1-limited access, or between 0.001 and 0.006 packets per node per slot, for exhaustive access. Traffic from class 1 nodes is scheduled immediately after the beacon, followed by traffic from class 2 nodes. Penalty coefficient for higher priority traffic (class 1 ) was fixed at $k_{p}^{(1)}=0.25$ while that for lower priority traffic (class 2) was varied between $k_{p}^{(1)}=0.25$ and 1.25 in five steps. The piconet uses $N=30 \mathrm{RF}$ channels with independent primary sources that are characterized by exponentially distributed $\mathrm{ON}$ and $\mathrm{OFF}$ periods with mean values of 900 and 2100 slots, respectively, resulting in mean cycle time of $T_{c y c}=3000$ slots with activity factor of $p_{\text {on }}=0.3$. We have assumed that data packets have a constant size of $k_{d}=10$ slots, while acknowledgment packets take a single slot. The superframe size was set to $s_{f}=100$ unit slots.

In our first experiment both traffic classes are serviced using gated exhaustive policy, hence the differentiation is achieved through sensing penalty only. Fig. 3 shows mean duration of service period, cycle time and waiting time for nodes in traffic classes 1 and 2, respectively. As can be seen, higher sensing penalty for class 2 traffic results in strong differentiation between various performance parameters such as node service time, piconet cycle time, and packet waiting time in particular. Scheduling higher priority traffic before the lower priority one has a moderate impact only, as witnessed by comparing the diagrams in the top row of Fig. 3 with the lines that correspond to $k_{p}=0.25$ in the diagrams in the bottom row.

In the same setting, total collision probabilities for both traffic classes as well as mean number of sensing nodes, mean interval between sensing events and probability of errors in the 


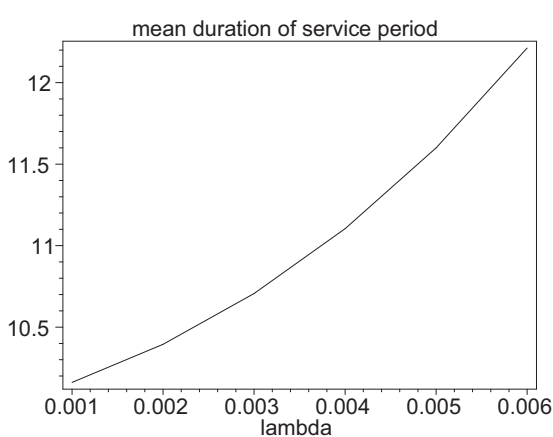

(a) Mean service period for class 1 traffic.

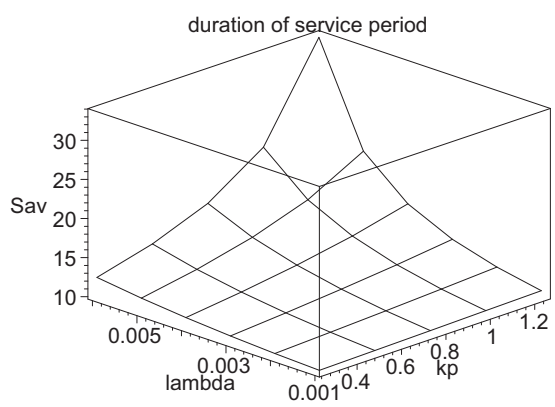

(d) Mean service period for class 2 traffic.

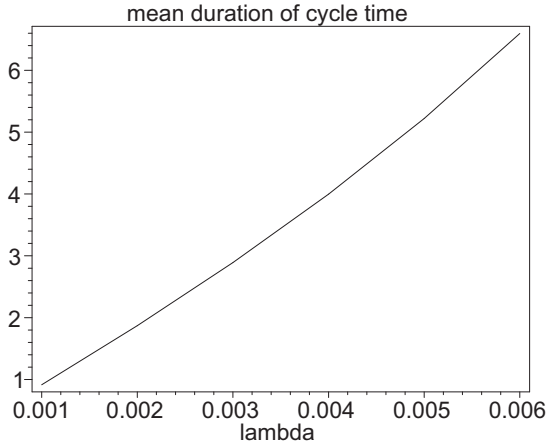

(b) Mean piconet cycle time for class 1 traffic.

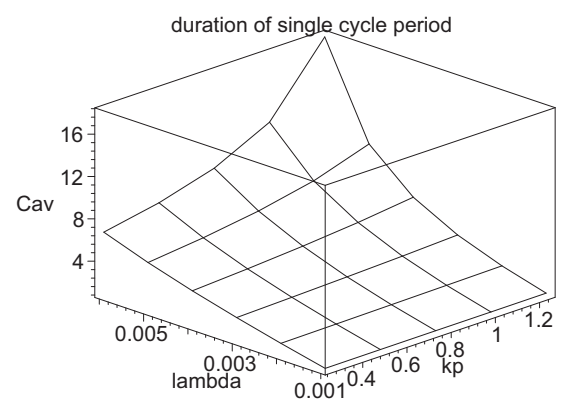

(e) Mean piconet cycle time for class 2 traffic.

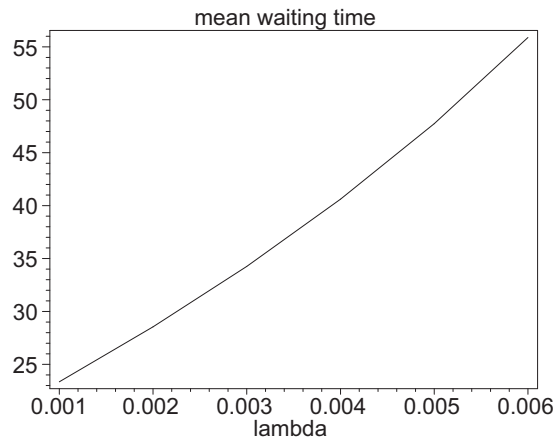

(c) Mean packet waiting time for class 1 traffic

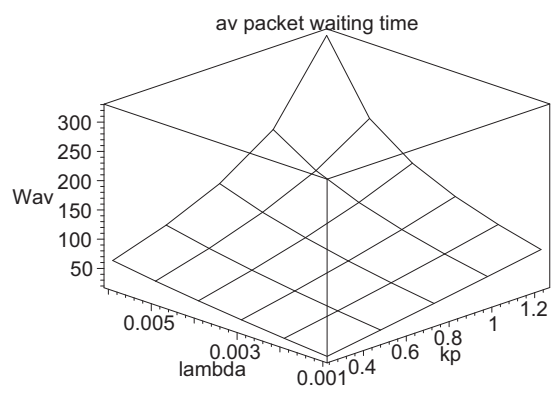

(f) Mean packet waiting time for class 2 traffic.

Fig. 3. Priority differentiation under variable sensing penalty for class 2 traffic (both classes use exhaustive service).

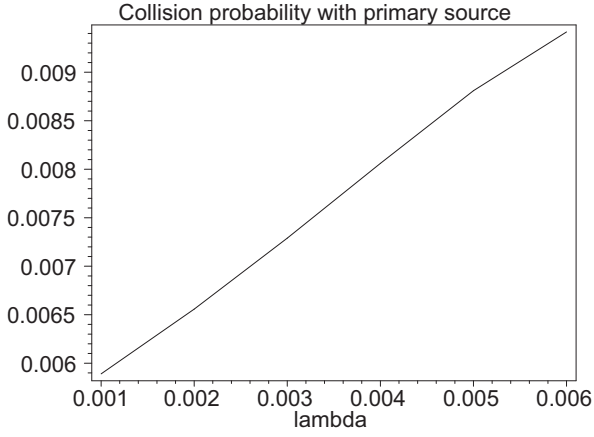

(a) Collision probability for traffic class 1 .

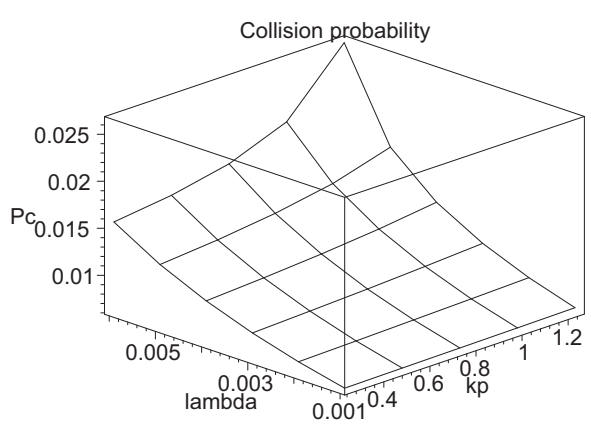

(b) Collision probability for traffic class 2 .

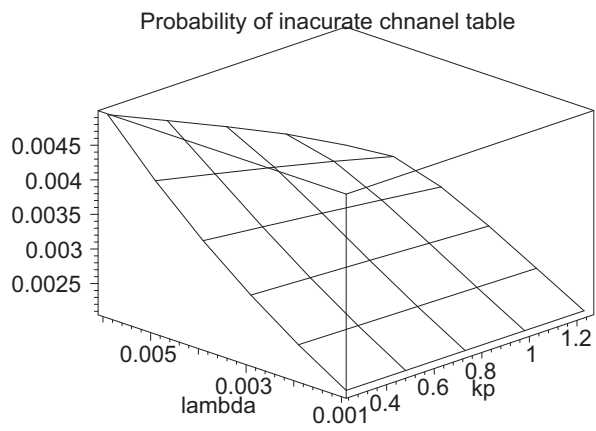

(c) Collision probability due to inaccurate channel table.

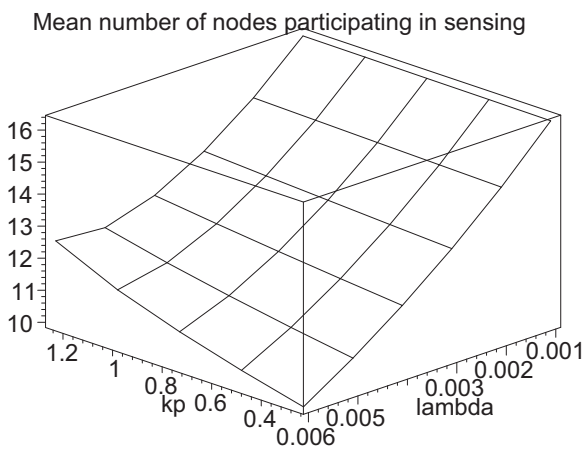

(d) Mean number of sensing nodes.

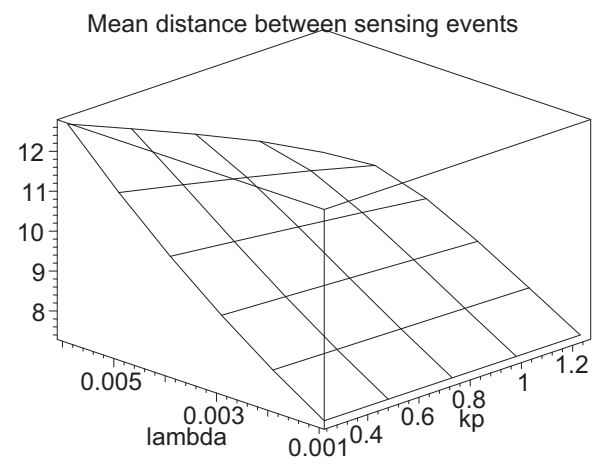

(e) Mean interval between sensing events on a channel.

Fig. 4. Sensing and collision descriptors under variable sensing penalty for class 2 traffic (both classes use exhaustive service). 


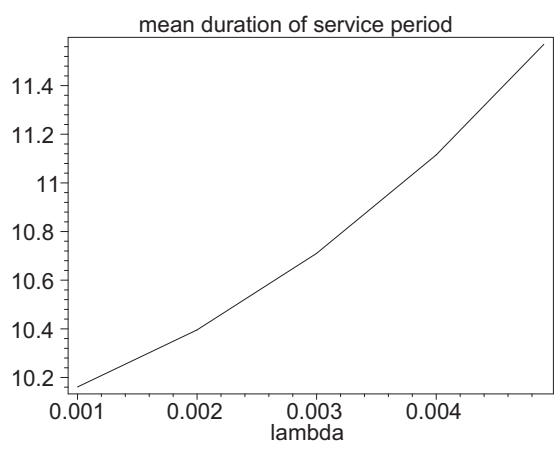

(a) Mean service period for class 1 traffic.

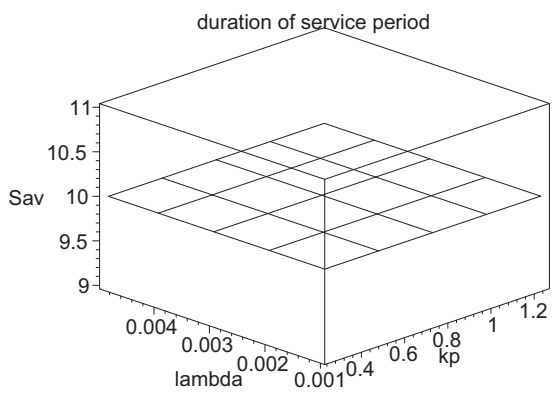

(d) Mean service period for class 2 traffic.

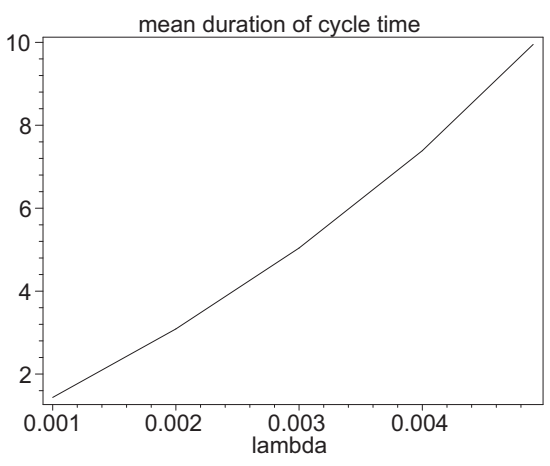

(b) Mean piconet cycle time for class 1 traffic.

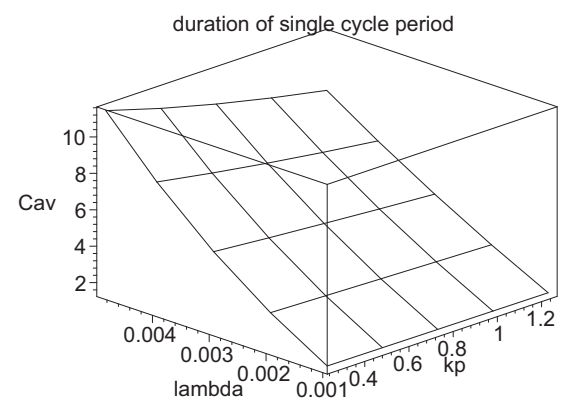

(e) Mean piconet cycle time for class 2 traffic.

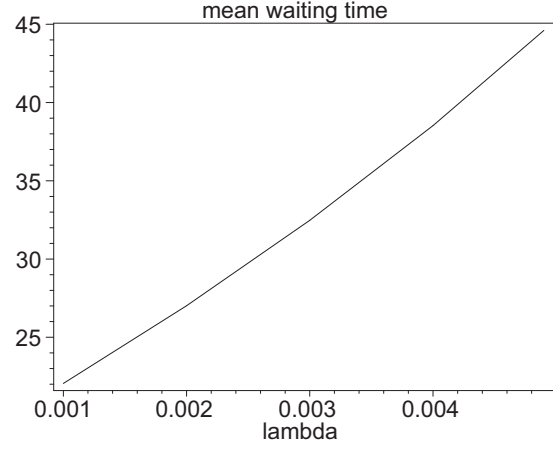

(c) Mean packet waiting time for class 1 traffic.

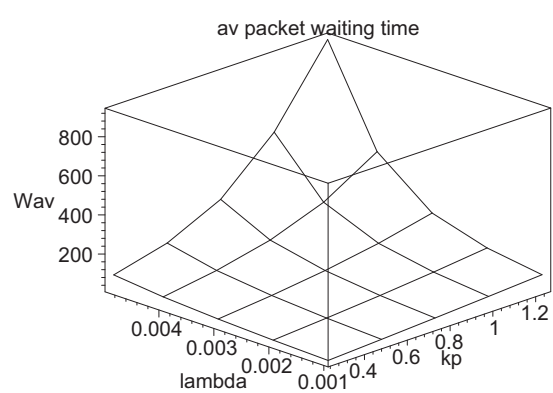

(f) Mean packet waiting time for class 2 traffic.

Fig. 5. Priority differentiation under exhaustive service for class 1 traffic, 1-limited service and variable sensing penalty for class 2 traffic.

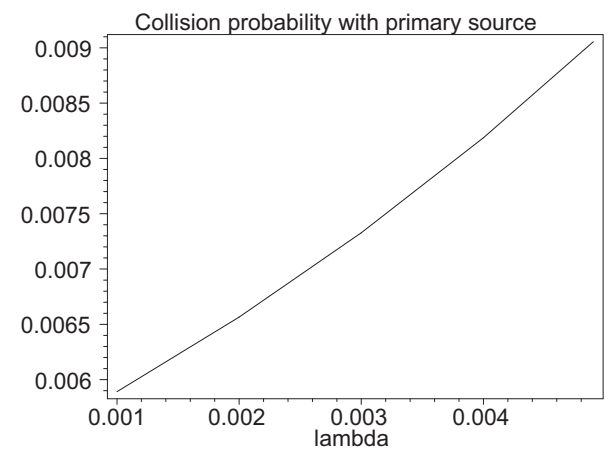

(a) Collision probability for traffic class 1 .

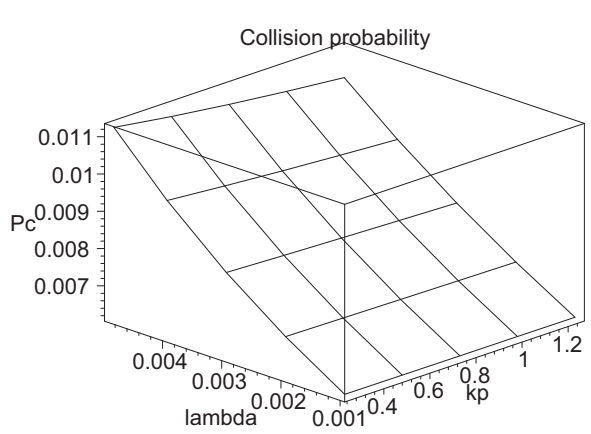

(b) Collision probability for traffic class 2 .

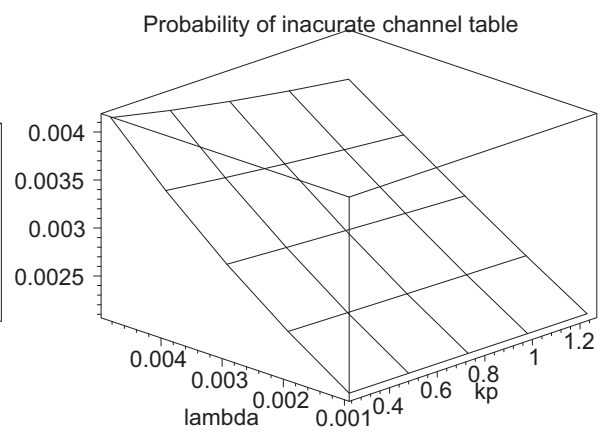

(c) Collision probability due to inaccurate channel table.

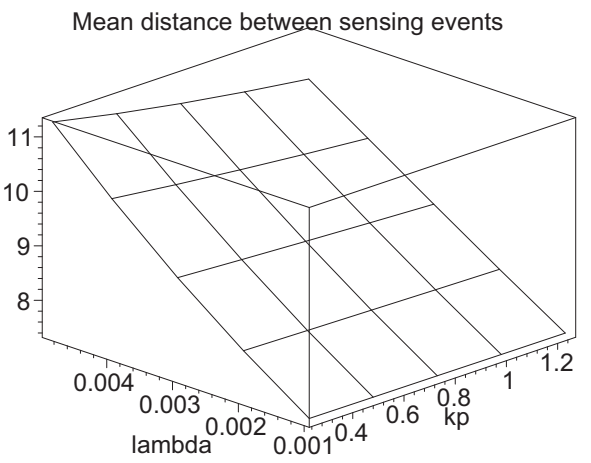

(e) Mean interval between sensing events on a channel.

Fig. 6. Sensing and collision descriptors under exhaustive service for class 1 traffic, 1-limited service and variable sensing penalty for class 2 traffic. 
channel table are shown in Fig. 4. At low packet arrival rate, nodes perform a lot of sensing and penalty coefficient has little impact. At higher traffic load, nodes spend less time sensing and collision probability increases together with the errors in the channel table. As the result, more sensing is needed that can be provided only by class 2 nodes, through an increase in the sensing penalty coefficient. While the entire network benefits from improved sensing accuracy, it is only class 2 nodes that bear the burden, through much higher packet delay times and higher collision probability caused by longer periods of transmission.

In our second experiment we have used gated exhaustive service for class 1 traffic and gated 1-limited service for class 2 traffic; furthermore, the sensing penalty for class 2 nodes was varied as above. Fig. 5 shows mean duration of service period, cycle time and waiting time for nodes in traffic classes 1 and 2, respectively. We observe that priority differentiation is much stronger than in previous case, in particular with respect to the packet waiting time.

Fig. 6 shows sensing and collision descriptors. Due to the increased overhead per packet caused by 1-limited service (where each packet is separately serviced), the network capacity has actually decreased which is why we have used reduced the maximum packet arrival rate to $\lambda_{1}=\lambda_{2}=0.005$ packets per node per slot. Class 2 nodes still provide the majority of sensing, but their contribution is not as efficient as under exhaustive access. As the result, class 1 experiences about $10 \%$ higher collision probability. However, the collision rate for class 2 traffic is somewhat reduced, thus partly compensating for larger packet delays.

The sensing mechanism performs very well under both service policies as long as the ratio of number of sensing nodes and number of channels exceeds 0.5 and the overall traffic load is small. Under higher traffic load, exhaustive scheduling is better for overall sensing reliability since the scheduling overhead is lower and more time can be devoted to sensing. Waiting times for class 2 packets are also lower compared to 1-limited scheduling, but class 2 packets suffer more from collisions with transmissions.

\section{CONCLUSION}

We have described and evaluated priority differentiation in a cognitive personal area network using different service policies and/or variable sensing penalty. Exhaustive scheduling provides better control of sensing and fewer collisions for both traffic classes. However, these results don't come for free: under exhaustive scheduling, increasing the sensing penalty for lower priority traffic leads to an increase in both delays and collision probability. Under 1-limited scheduling, increasing the sensing penalty for lower priority traffic leads to much high packet delays for lower priority traffic but also to increased collision probability for higher priority traffic.
In future we will experiment with other service policies and try to optimize the tradeoff between collision and delay performance for both traffic classes, and extend our work to more than two traffic classes as well.

\section{REFERENCES}

[1] A. Alshamrani, X. Shen, and L.-L. Xie. QoS provisioning for heterogeneous services in cooperative cognitive radio networks. IEEE $J$. on Selected Areas in Communications - Wireless Series, 29(4):819-830, 2011.

[2] A. Attar, M. R. Nakhai, and A. H. Aghvami. Cognitive radio game: a framework for efficiency, fairness and QoS guarantee. In Proc. IEEE International Conference on Communications ICC 2008, pages 41704174, Beijing, China, May 2008.

[3] L. Cai, Y. Liu, X. Shen, J. W. Mark, and D. Zhao. Distributed QoS-aware MAC for multimedia over cognitive radio networks. In Proc. Global Telecommunications Conference GLOBECOM'10, pages 1-5, Miami, FL, Dec. 2010.

[4] S. Chakrabarti and A. Mishra. QoS issues in ad hoc wireless networks. IEEE Communications Magazine, 39(2):142-148, 2001.

[5] J. Chen, M. Zhao, J. Tian, and S. Li. A QoS MAC protocol for cognitive PMP networks with rapid changes of spectrum opportunities. In IEEE Vehicular Technology Conference (VTC-Spring 2008), pages 1661-1665, Singapore, May 2008.

[6] K. Hamdi, W. Zhang, and K. Letaief. Uplink scheduling with QoS provisioning for cognitive radio systems. In WCNC 2007, pages 25922596, Hong Kong, China, Mar. 2007.

[7] D. P. Heyman and M. J. Sobel. Stochastic Models in Operations Research, Volume I: Stochastic Processes and Operating Characteristics. McGraw-Hill, New York, 1982.

[8] B. Ishibashi, N. Bouabdallah, and R. Boutaba. QoS performance analysis of cognitive radio-based virtual wireless networks. In Proc. INFOCOM, Phoenix, AZ, Apr. 2008.

[9] H. Levy, M. Sidi, and O. J. Boxma. Dominance relations in polling systems. Queueing Systems Theory and Applications, 6(2):155-171, 1990.

[10] Maplesoft, Inc. Maple 16. Waterloo, ON, Canada, 2012.

[11] J. Mišić and V. B. Mišić. Performance of cooperative sensing at the MAC level: Error minimization through differential sensing. IEEE Transactions on Vehicular Technology, 58(5):2457-2470, June 2009.

[12] J. Mišić and V. B. Mišić. Recovery in channel-hopping cognitive networks under random primary user activity. IEEE Transactions on Vehicular Technology, 63(5):2392-2406, June 2014.

[13] S. Shankar, C.-T. Chou, K. Challapali, and S. Mangold. Spectrum agile radio: capacity and QoS implications of dynamic spectrum assignment. In Proc. Global Telecommunications Conference GLOBECOM'05, volume 5, pages 2510-2516, St. Louis, MO, Nov. 2005.

[14] H. Su and X. Zhang. Cross-layer based opportunistic MAC protocols for $\mathrm{QoS}$ provisionings over cognitive radio wireless networks. IEEE J. on Selected Areas in Communications - Wireless Series, 26(1):118-129, 2008.

[15] H. Takagi. Queueing Analysis, volume 1: Vacation and Priority Systems. North-Holland, Amsterdam, The Netherlands, 1991.

[16] L.-C. Wang, A. Chen, and D. S. L. Wei. A cognitive MAC protocol for QoS provisioning in overlaying ad hoc networks. In CCNC 2007, pages 1139-1143, Las Vegas, NV, Jan. 2007.

[17] L.-C. Wang, C.-W. Wang, and F. Adachi. Load-balancing spectrum decision for cognitive radio networks. IEEE J. on Selected Areas in Communications - Wireless Series, 29(4):757-769, Apr. 2011.

[18] L.-C. Wang, C.-W. Wang, and K.-T. Feng. A queueing-theoretical framework for QoS-enhanced spectrum management in cognitive radio networks. IEEE Wireless Communications, 18(6):18-26, Dec. 2011.

[19] Q. Zhao, S. Geirhofer, L. Tong, and B. M. Sadler. Optimal dynamic spectrum access via periodic channel sensing. In IEEE Wireless Communications and Networking Conference (WCNC2007), pages 3337, Hong Kong, China, Mar. 2007. 